\title{
Inhibitory activities of selected Sudanese medicinal plants on Porphyromonas gingivalis and matrix metalloproteinase- 9 and isolation of bioactive compounds from Combretum hartmannianum (Schweinf) bark
}

Ebtihal Abdalla M. Mohieldin ${ }^{1,2}$, Ali Mahmoud Muddathir ${ }^{3^{*}}$ and Tohru Mitsunaga ${ }^{2}$

\begin{abstract}
Background: Periodontal diseases are one of the major health problems and among the most important preventable global infectious diseases. Porphyromonas gingivalis is an anaerobic Gram-negative bacterium which has been strongly implicated in the etiology of periodontitis. Additionally, matrix metalloproteinases-9 (MMP-9) is an important factor contributing to periodontal tissue destruction by a variety of mechanisms. The purpose of this study was to evaluate the selected Sudanese medicinal plants against P. gingivalis bacteria and their inhibitory activities on MMP-9.

Methods: Sixty two methanolic and 50\% ethanolic extracts from 24 plants species were tested for antibacterial activity against $P$. gingivalis using microplate dilution assay method to determine the minimum inhibitory concentration (MIC). The inhibitory activity of seven methanol extracts selected from the 62 extracts against MMP-9 was determined by Colorimetric Drug Discovery Kit. In search of bioactive lead compounds, Combretum hartmannianum bark which was found to be within the most active plant extracts was subjected to various chromatographic (medium pressure liquid chromatography, column chromatography on a Sephadex LH-20, preparative high performance liquid chromatography) and spectroscopic methods (liquid chromatography-mass spectrometry, Nuclear Magnetic Resonance (NMR)) to isolate and characterize flavogalonic acid dilactone and terchebulin as bioactive compounds.

Results: About $80 \%$ of the crude extracts provided a MIC value $\leq 4 \mathrm{mg} / \mathrm{ml}$ against bacteria. The extracts which revealed the highest potency were: methanolic extracts of Terminalia laxiflora (wood; MIC $=0.25 \mathrm{mg} / \mathrm{ml}$ ) followed by Acacia totrtilis (bark), Ambrosia maritima (aerial part), Argemone mexicana (seed), C. hartmannianum (bark), Terminalia brownii (wood) and $50 \%$ ethanolic extract of T. brownii (bark) with MIC values of $0.5 \mathrm{mg} / \mathrm{ml}$. T. laxiflora (wood) and C. hartmannianum (bark) which belong to combretaceae family showed an inhibitory activity over $50 \%$ at the concentration of $10 \mu \mathrm{g} / \mathrm{ml}$ against MMP-9. Additionally, MMP-9 was significantly inhibited by terchebulin with $I_{50}$ value of $6.7 \mu \mathrm{M}$.

(Continued on next page)
\end{abstract}

\footnotetext{
*Correspondence: a_muddathir@yahoo.com; muddathir@uofk.edu

${ }^{3}$ Department of Horticulture, Faculty of Agriculture, University of Khartoum,

Khartoum North-Shambat, Sudan

Full list of author information is available at the end of the article
} 
(Continued from previous page)

Conclusions: To the best of our knowledge, flavogalonic acid dilactone and terchebulin were isolated from $C$. hartmannianium bark for the first time in this study. Because of terchebulin and some crude extracts acting on $P$. gingivalis bacteria and MMP-9 enzyme that would make them promising natural preference for preventing and treating periodontal diseases.

Keywords: Sudanese medicinal plants, Combretum hartmannianum, Porphyromonas gingivalis, MMP-9, Flavogalonic acid dilacton, Terchebulin

\section{Background}

Periodontal diseases are multifactorial infections caused by a specific group of Gram-negative anaerobic bacteria leading to destruction of the tooth-supporting tissue including the alveolar bone and the periodontal ligament. Two major factors contributed to the pathogenesis of periodontitis are namely periodontopathogens which cause direct damage to periodontal tissue through the secretion of toxic products, and the host response to periodontopathogens which results in the release of inflammatory mediators (proinflammatory cytokines, matrix metalloproteinases (MMPs) and prostanoids) [1].

Porphyromonas gingivalis, a Gram-negative, black pigmented and an anaerobic bacterium, has been strongly implicated in the etiology of some types of periodontitis including chronic adult periodontitis [2, 3]. It is a major periodontal pathogen that possesses multiple virulence factors including gingipains, lipopolysaccharides and can trigger host cells to release inflammatory cytokines and MMPs [4]. Previous studies showed that MMP-9 secretions were unregulated by $P$. gingivalis supernatant in periodontal ligament fibroblasts, pulp fibroblasts and osteosarcoma cells [5-7].

Most of the Sudanese people in rural areas rely on traditional medicine for the treatment of many infectious diseases (Table 1). Different plant species of medicinal importance have successfully been included in mouthwashes and toothpastes in many countries [8-10]. Human pathogenic microorganisms have developed resistance to drugs owing to the extensive often use of commercial synthetic antibacterial drugs in large quantities without proper medical prescriptions and tests. This condition has raised alarm in most countries and scientists are forced to search for an alternative to these compounds, often in the form of natural medicines from sources such as plants [11].

Combretum hartmannianum a shrub up to $4 \mathrm{~m}$; as a tree under favorable conditions $10 \mathrm{~m}$ high. The plant is widespread throughout the Sahel belt from Senegal to Cameroon, and eastwards to the Sudan [12]. Leaves, fruits and stem bark extracts of $C$. hartmannianum showed activity against Gram-positive bacteria, E. coli (Gram-negative); and have also been reported to exhibit anti-inflammatory activity $[13,14]$.
Hence, the purpose of this study was firstly to investigate the antibacterial activity against $P$. gingivalis bacteria of 62 methanolic and 50\% ethanolic extracts from 24 selected Sudanese medicinal plants species. Secondly, from these 62 extracts; seven methanol extracts (Terminalia laxiflora, Tamarix nilotica, Khaya senegalensis, Acacia seyal var. fistula, Acacia seyal var. seyal, C. hartmannianum and Terminalia brownii) were selected to examine their inhibitory activities against MMP-9 enzyme. Additionally methanolic extract of $C$. hartmannianum bark that demonstrated good combined activities were subjected for further fractionation in order to identify the active compounds responsible for the biological activities.

\section{Methods}

\section{Plant materials}

Twenty four different plant species were collected from Khartoum and Elgadarif States, Sudan, identified and authenticated by Dr. Ashraf Mohamed from the Faculty of Forestry, Mrs. Hamza Tag EL-Sir Herbarium Curator. Voucher specimens (Table 3) were deposited in the Horticultural Laboratory, Department of Horticulture, Faculty of Agriculture, University of Khartoum.

\section{Preparation of plant extracts}

Different plant parts (Table 3) were dried under shade and then grounded before they were subjected to cold maceration with methanol or $50 \%$ ethanol. The plant powder was macerated with a gentle shaking for $12 \mathrm{~h}$ three times in solvents in side stoppered flasks at room temperature. The extracted solvents were filtrated and evaporated under reduced pressure using a rotatory evaporator, and the concentrated $50 \%$ ethanol extracts were then dried with a freeze dryer, resulting in 62 crude extracts and stored at $4{ }^{\circ} \mathrm{C}$ until use. In order to prepare stock solution, extracts were dissolved in 100\% dimethyl sulfoxide (DMSO). Further serial dilution of the stock was performed to obtain a range of desired concentration of the extracts.

\section{Fractionation, purification and isolation of Combretum hartmannianum bark}

Five grams of C. hartmannianum bark methanolic extract was subjected to fractionation by medium pressure 
Table 1 Selected Sudanese medicinal plant species used in traditional medicine

\begin{tabular}{|c|c|c|c|c|c|}
\hline No & Botanical names & Family & $\begin{array}{l}\text { Vernacular } \\
\text { name }\end{array}$ & Part used & Traditional uses \\
\hline 1 & $\begin{array}{l}\text { Calotropis procera (Aiton) } \\
\text { Dryand }\end{array}$ & Apocynaceae & Ushar & Leaves & $\begin{array}{l}\text { Fever, joint pains, muscular spasm, constipation, against } \\
\text { scorpion bites, jaundice [45], healing thorn injuries [46], } \\
\text { anti-rheumatic }[47,48] .\end{array}$ \\
\hline 2 & Arestolochia bracteolate Lam. & Aristochiaceae & Um- Galagel & Whole plant & Malaria, HIV-1 $[49,50]$. \\
\hline 3 & Xanthium brasilicum Vell. & Asteraceae & Ramtouk & Leaves & Venereal diseases, malaria $[51,52]$ \\
\hline 4 & Vernonia amygdalina Delile & & Gharib elwadi & Leaves & Fever, gastro-intestinal disease "GID" [53]. \\
\hline 5 & Adanosonia digitata $\mathrm{L}$. & Bombacaceae & Tabaldi & Fruit pulp & $\begin{array}{l}\text { The fruits are used as a cold beverage, added to yoghurt } \\
\text { for treatment of diarrhea and amoebic dysentery [54]. }\end{array}$ \\
\hline 6 & Terminalia laxiflora Engl. & Combretaceae & Darut & Wood & Malaria, cough treatments, heartwood for fumigant $[55,56]$. \\
\hline 7 & Terminalia brownii Fresen & & Sobagh, Shaff & Wood, bark & Against cough and bronchitis [47], anti-rheumatic [48]. \\
\hline 8 & $\begin{array}{l}\text { Combretum hartmannianum } \\
\text { (Schweinf) }\end{array}$ & & Habil & Wood, bark & Febrile, jaundice, bacterial infections $[37,38]$. \\
\hline 9 & Ambrosia maritima L. & Compositae & Damsisa & Aerial part & $\begin{array}{l}\text { The herbs are used in treatment of urinary tract infections } \\
\text { and elimination of kidney stones, whereas the leaves are } \\
\text { used as anti-diabetic and anti-hypertensive [57]. }\end{array}$ \\
\hline 10 & Euphorbia hirta L. & Euphorbiaceae & Um libina & Aerial part & Decoction of plant is use in asthma and bronchitis [58]. \\
\hline 11 & Ricinus communis L. & & Khirwe & Leaves & $\begin{array}{l}\text { The leaves are used as a poultice in treatment of abscesses } \\
{[59,60] \text {. }}\end{array}$ \\
\hline 12 & $\begin{array}{l}\text { Acacia seyal var. fistula } \\
\text { (Schweinf.) }\end{array}$ & Leguminosae & Sfar abide & Wood, bark & Fumigation, rheumatic pain [61]. \\
\hline 13 & Acacia seyal var. seyal Del. & & Talih & Wood, bark & Anti-rheumatic, mouth detergent [62]. \\
\hline 14 & Acacia tortilis (Forssk.) Hayne & & Seyyal & Wood, bark & Treat skin infection, allergic dermatomes [63]. \\
\hline 15 & Cassia acutifolia Delile & & Senna makka & Leaves & Laxative [64], against GID [48]. \\
\hline 16 & Parkinsonia aculeata L. & & Sesaban & Leaves & Antipyretic, anti- diabetics [63]. \\
\hline 17 & Senna italica Mill. & & Sin elkalb & Leaves & $\begin{array}{l}\text { Intestinal complications, haemomorphoids, circulatory system } \\
\text { problems, calculi in the urinary system, sexually transmitted } \\
\text { diseases [65]. }\end{array}$ \\
\hline 18 & $\begin{array}{l}\text { Khaya senegalensis (Desv) A. } \\
\text { Juss }\end{array}$ & Meliaceae & Mahogany & Bark & $\begin{array}{l}\text { Anti-malarial, against hepatic inflammation, sinusitis, skin } \\
\text { diseases, GID, trachoma [48]. }\end{array}$ \\
\hline 19 & Polygonum glabrum Willd & Polygonaceae & Altomsahia & Leaves & Anthelminthic, antimalarial [66]. \\
\hline 20 & Argemone mexicana $\mathrm{L}$. & Papaveraceae & Argemone & Leaves, seed & Venereal diseases [52]. \\
\hline 21 & Solanum dubium Fresen & Solanaceae & Gibben & Fruits & $\begin{array}{l}\text { The whole plant and fruits are pulped and applied to wounds } \\
\text { and skin tumors as a dressing [67]. }\end{array}$ \\
\hline 22 & Salvadora persica $\mathrm{L}$. & Salvadoraceae & Alarak & Leaves, stem & Gingivitis, malaria liver swellings, HIV-1 $[50,68,69]$. \\
\hline 23 & Tamarix nilotica (Ehrenb.)Bunge & Tamaricaceae & Tarft al nil & Stem & Febrile, colds [69]. \\
\hline 24 & Tribulus terrestri $\mathrm{L}$. & Zygophyllaceae & Derresia & Aerial part & Demulcent, renal nephritis [47]. \\
\hline
\end{tabular}

liquid chromatography (MPLC) using ODS column (YMCDispoPack AT ODS-25:120 g). The column was conditioned with the first eluent used for separation for $30 \mathrm{~min}$ with flow rate $0.5 \mathrm{ml} / \mathrm{min}$. MPLC separation was performed by using a chromatography pump (540 Yamazen, Japan), UV detector at $280 \mathrm{~nm}$ wavelength (UV-10 V Yamazen, Japan) and a fraction collector (SF-2120, Advantec Tokyo Ltd., Japan). Elution with $\mathrm{H}_{2} \mathrm{O} / \mathrm{MeOH} 95 / 5,20$ / 80 and absolute methanol resulted in three fractions (F1, F2 and F3). Fraction one (F1), which demonstrated a good inhibitory activity against bacteria and enzyme, was subjected to column chromatography on a Sephadex LH-20 eluted with methanol (90-20\%) in water, and finally washed with $70 \%$ acetone to give five sub-fractions. Separation of these sub-fractions mainly, (F1-1, F1-2 and F1-3) were performed by using preparative high performance liquid chromatography (HPLC) with reversed phase Inertsil ODS-3 column (GL Sciences Inc. $10 \mathrm{~mm}$ i.d. $\times 250 \mathrm{~mm}$ ) monitored at $280 \mathrm{~nm}$. The solvent system used was as follows: a gradient program for $60 \mathrm{~min}$ from 10 to $100 \%$ methanol in water with $0.05 \%$ TFA at a flow rate $5 \mathrm{ml} / \mathrm{min}$ [15].

Compounds were identified by liquid chromatographymass spectrometry (LC-MS) with negative ion mode and ${ }^{1} \mathrm{H},{ }^{13} \mathrm{C}$ NMR. Methanol-d4 was used as the NMR solvent. NMR measurements were obtained by using JEOL ECP 
Table $2{ }^{1} \mathrm{H}$ - and ${ }^{13} \mathrm{C}-\mathrm{NMR}$ data of flavogallonic acid dilactone and terchebulin (in $\mathrm{CD}_{3} \mathrm{OD}$ ) as compared with literature [16, 17]

\begin{tabular}{|c|c|c|c|c|c|c|c|c|c|}
\hline \multirow[t]{2}{*}{ Position } & \multicolumn{4}{|c|}{ Flavogallonic acid dilactone } & & \multicolumn{4}{|l|}{ Terchebulin } \\
\hline & $\begin{array}{l}{ }^{1} \mathrm{H}(\mathrm{ppm}) \mathrm{JH}, \mathrm{H} \\
(\mathrm{Hz})\end{array}$ & $\begin{array}{l}{ }^{1} \mathrm{H}(\mathrm{ppm}) \\
{[16]}\end{array}$ & $\begin{array}{l}{ }^{13} \mathrm{C} \\
\text { (ppm) }\end{array}$ & $\begin{array}{l}{ }^{13} \mathrm{C}(\mathrm{ppm}) \\
{[16]}\end{array}$ & & ${ }^{1} \mathrm{H}(\mathrm{ppm}) \mathrm{JH}, \mathrm{H}(\mathrm{Hz})$ & ${ }^{1} \mathrm{H}$ (ppm) [17] & $\begin{array}{l}{ }^{13} \mathrm{C} \\
\text { (ppm) }\end{array}$ & $\begin{array}{l}{ }^{13} \mathrm{C} \\
(\mathrm{ppm})[17]\end{array}$ \\
\hline & & & & & & & & 113.0 & \\
\hline & & & & & $A$ & & & 125.1 & \\
\hline \multirow[t]{5}{*}{1} & & & 108.1 & 107.3 & $B$ & & & 123.5 & \\
\hline & & & & & $C$ & & & 123.5 & \\
\hline & & & & & $\mathrm{D}$ & & & 122.2 & \\
\hline & & & & & & & & 114.0 & \\
\hline & & & & & $A$ & $6.56(\mathrm{~S}, \mathrm{H})$ & $6.63(\mathrm{~s}, \mathrm{H})$ & 106.8 & \\
\hline \multirow[t]{5}{*}{2} & & & 135.7 & 135.2 & B & & & 113.0 & \\
\hline & & & & & C & $6.79(\mathrm{~s}, \mathrm{H})$ & $6.80(\mathrm{~s}, \mathrm{H})$ & 108.5 & \\
\hline & & & & & $\mathrm{D}$ & & & 141.7 & \\
\hline & & & & & & & & 138.4 & \\
\hline & & & & & $A$ & & & 144.5 & \\
\hline \multirow[t]{5}{*}{3} & & & 136.3 & 136.2 & B & & & 143.4 & \\
\hline & & & & & $C$ & & & 144.6 & \\
\hline & & & & & $\mathrm{D}$ & & & 139.1 & \\
\hline & & & & & & & & 150.3 & \\
\hline & & & & & A & & & 136.1 & \\
\hline \multirow[t]{5}{*}{4} & & & 136.5 & 136.7 & B & & & 135.9 & \\
\hline & & & & & $C$ & & & 137.5 & \\
\hline & & & & & $\mathrm{D}$ & & & 137.6 & \\
\hline & & & & & & & & 113.0 & \\
\hline & & & & & $A$ & & & 143.4 & \\
\hline \multirow[t]{5}{*}{5} & $7.26(s)$ & $7.11(\mathrm{~s})$ & 112.8 & 111.3 & B & $7.48(\mathrm{~s}, \mathrm{H})$ & $7.58(\mathrm{~s}, \mathrm{H})$ & 144.5 & \\
\hline & & & & & $\mathrm{D}$ & & & 144.6 & \\
\hline & & & & & C & & & 143.6 & \\
\hline & & & & & & & & 114.0 & \\
\hline & & & & & A & & & 112.0 & \\
\hline \multirow[t]{4}{*}{6} & & & 110.1 & 109.5 & $B$ & $6.37(\mathrm{~s}, \mathrm{H})$ & $6.37(\mathrm{~s}, \mathrm{H})$ & 106.4 & \\
\hline & & & & & $C$ & & & 116.0 & \\
\hline & & & & & $D$ & $6.42(\mathrm{~s}, \mathrm{H})$ & $6.39(\mathrm{~s}, \mathrm{H})$ & 106.5 & \\
\hline & & & & & & & & 159.5 & 160.3 \\
\hline \multirow[t]{4}{*}{7} & & & & & $A$ & & & 168.9 & 169.9 \\
\hline & & & 158.9 & 157.1 & B & & & 169.5 & 169.1 \\
\hline & & & & & $C$ & & & 167.0 & 167.6 \\
\hline & & & & & $\mathrm{D}$ & & & 166.9 & 166.8 \\
\hline $1^{\prime}$ & & & 108.1 & 107.3 & & & & 112.0 & \\
\hline $2^{\prime}$ & & & 137.8 & 137.8 & & & & 114.0 & \\
\hline $3^{\prime}$ & & & 139.2 & 138.9 & & & & 140.7 & \\
\hline $4^{\prime}$ & & & 143.2 & 143.1 & & & & 147.4 & \\
\hline $5^{\prime}$ & & & 117.5 & 117.6 & & & & 113.0 & \\
\hline $6^{\prime}$ & & & 114.4 & 109.9 & & & & 114.0 & \\
\hline $7^{\prime}$ & & & 160.4 & 158.9 & & & & 158.3 & 157.9 \\
\hline
\end{tabular}


Table $2{ }^{1} \mathrm{H}$ - and ${ }^{13} \mathrm{C}-\mathrm{NMR}$ data of flavogallonic acid dilactone and terchebulin (in $\mathrm{CD}_{3} \mathrm{OD}$ ) as compared with literature [16, 17] (Continued)

\begin{tabular}{|c|c|c|c|c|c|c|c|c|}
\hline$\overline{1^{\prime \prime}}$ & & & 124.9 & 125.8 & $5.23(\mathrm{~d}, J=2.8 \mathrm{~Hz})$ & $5.32(\mathrm{~d}, J=4 \mathrm{~Hz})$ & 90.2 & 90.8 \\
\hline $2^{\prime \prime}$ & & & 120.2 & 120.0 & $4.98(\mathrm{dd}, J=3.5,9.7 \mathrm{~Hz})$ & $\begin{array}{l}4.88(\mathrm{dd} \\
J=3.9 \mathrm{~Hz})\end{array}$ & 74.2 & 75.1 \\
\hline $3^{\prime \prime}$ & & & 144.1 & 143.1 & $5.64(\mathrm{t}, J=9.6 \mathrm{~Hz})$ & $5.59(\mathrm{t}, J=8 \mathrm{~Hz})$ & 74.1 & 74.6 \\
\hline $4^{\prime \prime}$ & & & 145.9 & 145.8 & $4.78(\mathrm{t}, J=11.0 \mathrm{~Hz})$ & & 68.5 & 70.1 \\
\hline $5^{\prime \prime}$ & & & 147.8 & 147.5 & $4.21(\mathrm{t}, J=10.3 \mathrm{~Hz})$ & & 69.0 & 69.6 \\
\hline $6^{\prime \prime}$ & $7.50(\mathrm{~s})$ & $7.49(\mathrm{~s})$ & 113.3 & 112.5 & $\begin{array}{l}3.04(\mathrm{t}, J=11.6 \mathrm{~Hz}) 4.48 \\
(\mathrm{t}, J=8.9 \mathrm{~Hz})\end{array}$ & $3.10(\mathrm{~d}, J=12 \mathrm{~Hz})$ & 63.4 & 64.4 \\
\hline $7^{\prime \prime}$ & & & 168.9 & 167.1 & - & - & - & - \\
\hline
\end{tabular}

$600 \mathrm{MHz}$ NMR. Spectroscopic data of flavogalonic acid dilactone and terchebulin were in good correlation to published data $[16,17]$ (Table 2).

Determination of minimum inhibitory concentration (MIC) MIC was determined by the broth dilution method according to Iwaki et al. [18]. Prophyromonas gingivalis ATTC 33277 was cultured in a Brain-Heart Infusion broth supplemented with $0.5 \mu \mathrm{g} / \mathrm{ml}$ vitamin $\mathrm{K}$ and $5 \mu \mathrm{g} /$ $\mathrm{ml}$ hemin. The crude extracts and pure compounds were tested for antibacterial activity in sterile 96-well plates. The inoculums were prepared by diluting the broth culture to approximately $10^{8} \mathrm{cell} / \mathrm{ml}$. To each well; $100 \mu \mathrm{l}$ of microbial inoculums were added and followed by addition of media to achieve a final volume of $200 \mu \mathrm{l}$. The tested extracts or isolated compounds were prepared in a concentration range of 4000-31.3 $\mu \mathrm{g} / \mathrm{ml}$ using a two-fold dilution method. The experiments were performed in triplicate. Chlorhexidine was included in the assays as positive control. The cultures were incubated for $72 \mathrm{~h}$ at $37{ }^{\circ} \mathrm{C}$ under anaerobic conditions. Microbial growth was indicated after the addition of $50 \mu \mathrm{l}$ of $(0.2 \mathrm{mg} / \mathrm{ml}) \mathrm{p}$-iodonitrotetrazolium violet (INT) to the cultures and incubated at $37{ }^{\circ} \mathrm{C}$ for $2 \mathrm{~h}$. The MIC was defined as the lowest concentration that inhibited the color change of INT [19].

\section{Measurement of collagenase activity}

Collagenase (MMP-9) inhibition activities of seven selected methanolic extracts and isolated compounds were investigated by using a MMP-9 Colorimetric Drug Discovery Kit: AK-404, AK-414 and AK-412 (Enzo Life Science, Plymouth, PA, USA). Briefly, aliquots $(50 \mu \mathrm{l})$ of buffer solution were distributed into a 96 well plate. Twenty microliter of each diluted MMP-9, methanolic extracts or isolated compounds at different concentrations were added and reaction mixtures were incubated for $30 \mathrm{~min}$ at $37{ }^{\circ} \mathrm{C}$ and diluted substrate (thiopeptide; $10 \mu \mathrm{l})$ was added. N-Isobutyl-N-(4-methoxyphenylsulfonyl) glycyl hydroxamic acid (NNGH) was used as positive control. Inhibition was measured by continuously reading plates at absorbance $414 \mathrm{~nm}$ for $10 \mathrm{~min}$ in a microplate reader. All assays were performed independently in triplicate [20]. The inhibition of MMP-9 was calculated using the formula:

$$
\text { Inhibition } \%=[100-(\mathrm{VI} / \mathrm{VC})] \times 100
$$

Where:

VI: reaction velocity of (sample or inhibitor).

VC: reaction velocity of control.

\section{Statistical analysis}

The percentage and $\mathrm{IC}_{50}$ values of MMP-9 inhibitory activities were expressed as the mean value. The significant differences between extracts or isolated compounds were assessed by one-way analysis of variance (ANOVA) followed by pair wise comparison of the means using Tukey's multiple comparison test. Values were determined to be significant when $p$ was less than $0.05(p<0.05)$.

\section{Results and discussion}

In this study methanol and 50\% ethanol were chosen as solvent for extraction. As shown in the previous studies, nearly all of the identified components from plants active against microorganisms and enzyme may be related to the polyphenolic content of the plant extract, so the initial screenings of plants can be done by using crude aqueous or alcohol extraction [21, 22].

\section{Evaluation of MIC activity of plant extracts against P.gingivalis}

In our search for natural products with beneficial properties for oral health, we evaluated the ability of 24 Sudanese medicinal plants species belonging to 15 families in order to reveal the inhibitory activity against $P$.gingivalis bacteria. Botanical name, part used, voucher specimen and MIC activity of methanol and $50 \%$ ethanol extracts against $P$. gingivalis were shown in Table 3. Comparatively, methanol extracts displayed better anti- $P$. gingivalis activity than $50 \%$ ethanol extracts. Among 62 plant extracts; 50 extracts exhibited MIC activity at the concentration of $4 \mathrm{mg} / \mathrm{ml}$ or 
Table 3 Minimum inhibitory concentration (MIC) activities of selected Sudanese medicinal plants against $P$. gingivalis

\begin{tabular}{|c|c|c|c|c|}
\hline \multirow[t]{2}{*}{ Botanicals name } & \multirow[t]{2}{*}{ Examined part } & \multirow[t]{2}{*}{ Voucher specimen } & \multicolumn{2}{|c|}{$\mathrm{MIC} \mathrm{mg/ml}$} \\
\hline & & & $\mathrm{MeOH}$ & $50 \% \mathrm{EtOH}$ \\
\hline A. bracteolate Lam. & Whole plant & SD-SH-04 & 2 & 1 \\
\hline A. digitata $\mathrm{L}$. & Fruit pulp & SD-OD-27 & 2 & 4 \\
\hline A. maritima L. & Aerial part & SD-SH-03 & 0.5 & 2 \\
\hline \multirow[t]{2}{*}{ A. mexicana $\mathrm{L}$. } & Leaves & SD-KH-39 & - & $-{ }^{a}$ \\
\hline & Seed & & 0.5 & 2 \\
\hline \multirow[t]{2}{*}{ A. tortilis (Forssk.) Hayne } & Bark & SD-KH-07 & 0.5 & 4 \\
\hline & Wood & & - & 4 \\
\hline \multirow[t]{2}{*}{ A.seyal var. fistula (Schweinf.) } & Bark & SD-GF-06 & 1 & 4 \\
\hline & Wood & & - & - \\
\hline \multirow[t]{2}{*}{ A.seyal var. seyal Del. } & Bark & SD-GF-05 & 1 & 2 \\
\hline & Wood & & - & - \\
\hline \multirow[t]{2}{*}{ C. hartmannianum (Schweinf) } & Bark & SD-KH-04 & 0.5 & 1 \\
\hline & Wood & & 1 & - \\
\hline C. procera (Aiton) Dryand & Leaves & SD-SH-11 & 2 & 4 \\
\hline C.acutifolia Delile & Leaves & SD-SH-24 & 1 & 1 \\
\hline E. hirta L. & Aerial part & SD-SH-37 & 2 & 4 \\
\hline K. senegalensis (Desv) A. Juss & Bark & SD-SH-14 & 1 & 2 \\
\hline P. aculeata L. & Leaves & SD-SH-02 & 1 & 4 \\
\hline P. glabrum Willd & Leaves & SD-SH-A-03 & 1 & - \\
\hline R. communis $\mathrm{L}$. & Leaves & SD-SH-36 & 1 & 4 \\
\hline S. dubium Fresen & Fruits & SD-SH-34 & - & 2 \\
\hline S. italica Mill. & Leaves & SD-SH-25 & 2 & 2 \\
\hline \multirow[t]{2}{*}{ S. persica $\mathrm{L}$. } & Stem & SD-SH-09 & 1 & 4 \\
\hline & Leaves & & - & - \\
\hline \multirow[t]{2}{*}{ T. brownii Fresen } & Bark & SD-GF-02 & 1 & 0.5 \\
\hline & Wood & & 0.5 & 2 \\
\hline T. laxiflora Engl. & Wood & & 0.25 & 2 \\
\hline T. nilotica (Ehrenb.)Bunge & Stem & SD-OD-10 & 2 & 4 \\
\hline T. terrestri $\mathrm{L}$. & Aerial part & SD-SH-33 & 1 & 1 \\
\hline V. amygdalina Delile & Leaves & SD-KH-19 & 1 & 2 \\
\hline X. brasilicum Vell. & Leaves & SD-SH-12 & 2 & 2 \\
\hline
\end{tabular}

a:has no activity up to $4 \mathrm{mg} / \mathrm{ml}$, MeoH: Methanol, $50 \%$ EtOH: $50 \%$ Ethanol chlorohexidine as positive control has MIC value $0.0004 \mathrm{mg} / \mathrm{ml}$

less; moderate inhibitory activity $(\mathrm{MIC}=1 \mathrm{mg} / \mathrm{ml})$ were found in sixteen plant extracts. The most potent extracts were methanol extract of Terminalia laxiflora (MIC value $0.25 \mathrm{mg} / \mathrm{ml}$ ) followed by Ambrosia maritima, Argemone mexicana (seed), Terminalia brownii (wood), C. hartmannianum (bark), Acacia totrtilis (bark) and $50 \%$ ethanolic extract of T. brownii (bark) with MIC value $0.5 \mathrm{mg} / \mathrm{ml}$ (Table 3). According to the reported previous studies, T. laxiflora also showed potent antibacterial activity against Propionibacterium acne with MIC value $0.13 \mathrm{mg} / \mathrm{ml}$ and their activity was due to hydrolizable tannins [23].
Also noteworthy the combrataceae family; C. hartmannianum (bark), T. brownii (wood and bark) and T. laxiflora demonstrated inhibitory activity against $P$. gingivalis with MIC values $2 \mathrm{mg} / \mathrm{ml}$ or less, except $50 \%$ ethanol extract of C. hartmannianum (wood) had no activity up to $4 \mathrm{mg} / \mathrm{ml}$. This family has a wide range of tannins, flavonoids, terpenoids and stilbenoids [24, 25]. Flavonoids have been reported to be mainly active against Gramnegative bacteria [26]. In this study, methanolic extract of C. hartmannianum (bark) exhibited good activity against $P$. gingivalis (MIC $0.5 \mathrm{mg} / \mathrm{ml}$ ), and this was in agreement with Eldeen and Van [27] who reported that 


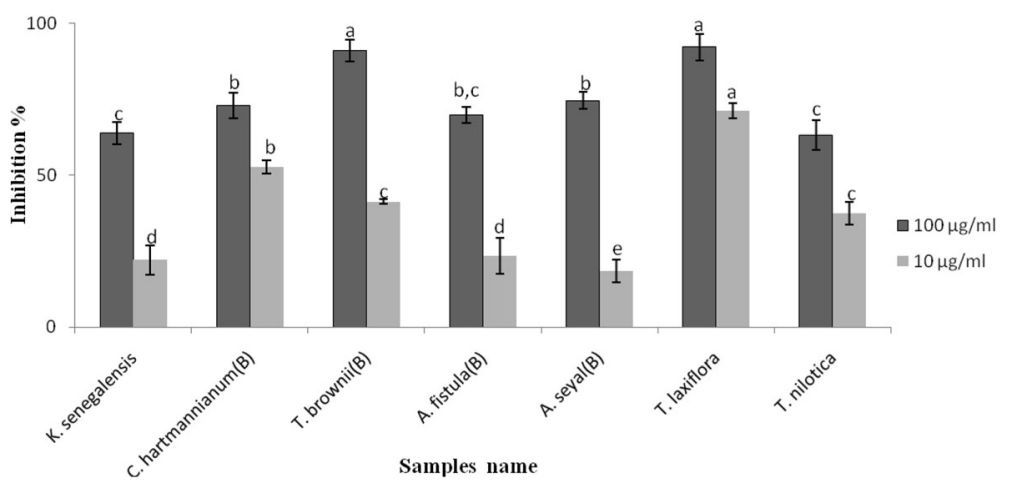

Fig. 1 Inhibitory activities of seven methanolic plants extracts against MMP-9. B: bark. NNGH as positive control has 100\% inhibition at concentration $100 \mu \mathrm{g} / \mathrm{ml}$. Values were expressed as mean $\pm \mathrm{SD}, n=3$. Values not followed by a common letter were significantly different at the level $(p<0.05)$

bark of C. hartmannianum inhibited the growth of Gram-negative bacteria at a concentration less than/or around $1.56 \mathrm{mg} / \mathrm{ml}$.

The positive control (chlorhexidine) showed a significant inhibitory activity compared to the other extracts. However, chlorhexidine has several side effects such as undesirable tooth discoloration, unpleasant taste and causing dryness and burning sensation in the mouth, leading to patient dissatisfaction $[28,29]$.

\section{Inhibitory activities of selected methanolic plants extracts against MMP-9}

Several therapeutic strategies, based on targeting different pathways of the pathogenesis of periodontal disease, have been put forward. In this regard, a number of authors proposed that periodontitis progression could be hampered by successfully inhibiting both bacteria and host-derived proteinases involved in connective tissue destruction of the periodontium [30, 31].

From our previous study to explore a natural agent for preventing and treatment of dental cavity, seven Sudanese methanolic extracts namely; T. laxiflora (wood), Tamarix nilotica (stem) and bark of Khaya senegalensis, Acacia seyal var. fistula, Acacia seyal var. seyal, C. hartmannianum and T. brownii showed potent inhibitory activity against glucosyltransferase enzyme that promotes the binding of cariogenic bacteria on the teeth (Additional file 1: Table S1). Therefore these seven methanolic extracts were selected for assayed their ability to inhibit the MMP-9 enzyme.

All seven selected extracts exhibited activity higher than $50 \%$ inhibition at the concentration of $100 \mu \mathrm{g} / \mathrm{ml}$ against MMP-9 (Fig. 1). NNGH (positive control) recorded $100 \%$ inhibition at the concentration of $100 \mu \mathrm{g} /$ $\mathrm{ml}$. At the concentration of $100 \mu \mathrm{g} / \mathrm{ml}$, methanolic extracts of $T$. laxiflora and $T$. brownii (bark) significantly inhibited MMP-9. Considerable, but less potent methanolic bark extracts of $A$. seyal var. seyal, C. hartmannianum and A. seyal var. fistula exhibited MMP-9 inhibitory activity at the concentration of $100 \mu \mathrm{g} / \mathrm{ml}$. Nevertheless, at the concentration $10 \mu \mathrm{g} / \mathrm{ml}$, T. laxiflora showed the potent inhibitory activity against MMP-9 followed by $C$. hartmannianum (bark). Kusumoto et al. [32] mentioned that the stem bark of Terminalia arjuna inhibited the HIV-1 protease activity by more than $70 \%$ at a concentration of $0.2 \mathrm{mg} / \mathrm{mL}$. Pomegranate methanol extract inhibited the secretion of MMP-9; this inhibitory effect was likely to be due to hydrolysable tannins [33]. Hydrolyzable tannins were suggested to exhibit their inhibitory effect on the tumor cell invasion via direct inhibition of MMP-9 activity [34]. Seigler [35] stated that Acacia spp. contained hydrolyzable tannins, flavonoids and condensed tannins.

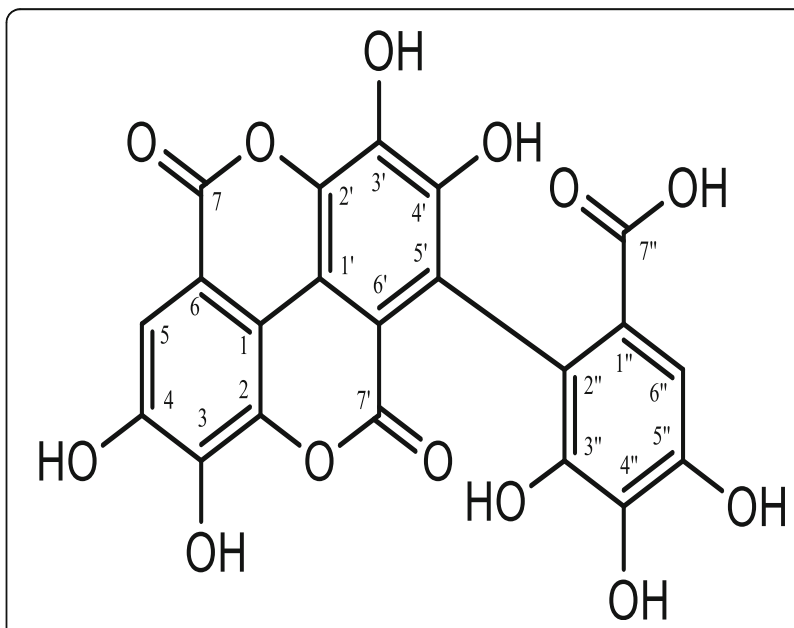

Fig. 2 Flavogallonic acid dilactone. Tan powder. LC-MS (negative ion mode) m/z: $469(\mathrm{M}-\mathrm{H})$ ); ${ }^{1} \mathrm{H}-\mathrm{NMR}$ (in $\mathrm{CD}_{3} \mathrm{OD}$ ): $\delta$ (ppm) 7.26 (s), 7.50 (s). ${ }^{13} \mathrm{C}-\mathrm{NMR}$ (in $\mathrm{CD}_{3} \mathrm{OD}$ ): $\delta$ (ppm) $108.1\left(\mathrm{C}-1,1^{\prime}\right), 110.1-114.4\left(\mathrm{C}-6,6^{\prime}\right)$, $112.8(C-5), 113.3\left(C-6^{\prime \prime}\right), 117.5-120.2\left(C-5^{\prime \prime}\right), 124.9\left(C-1^{\prime \prime}\right), 135.7(C-2)$, $136.3(\mathrm{C}-3), 136.5(\mathrm{C}-4), 137.8\left(\mathrm{C}-2^{\prime}\right), 139.2\left(\mathrm{C}-3^{\prime}\right), 143.2\left(\mathrm{C}-4^{\prime}\right), 144.1$ $\left(C-3^{\prime \prime}\right), 145.9\left(C-4^{\prime \prime}\right), 147.8\left(C-5^{\prime \prime}\right), 158.9-160.4\left(C-7,7^{\prime}\right), 168.9\left(C-7^{\prime \prime}\right)$ 


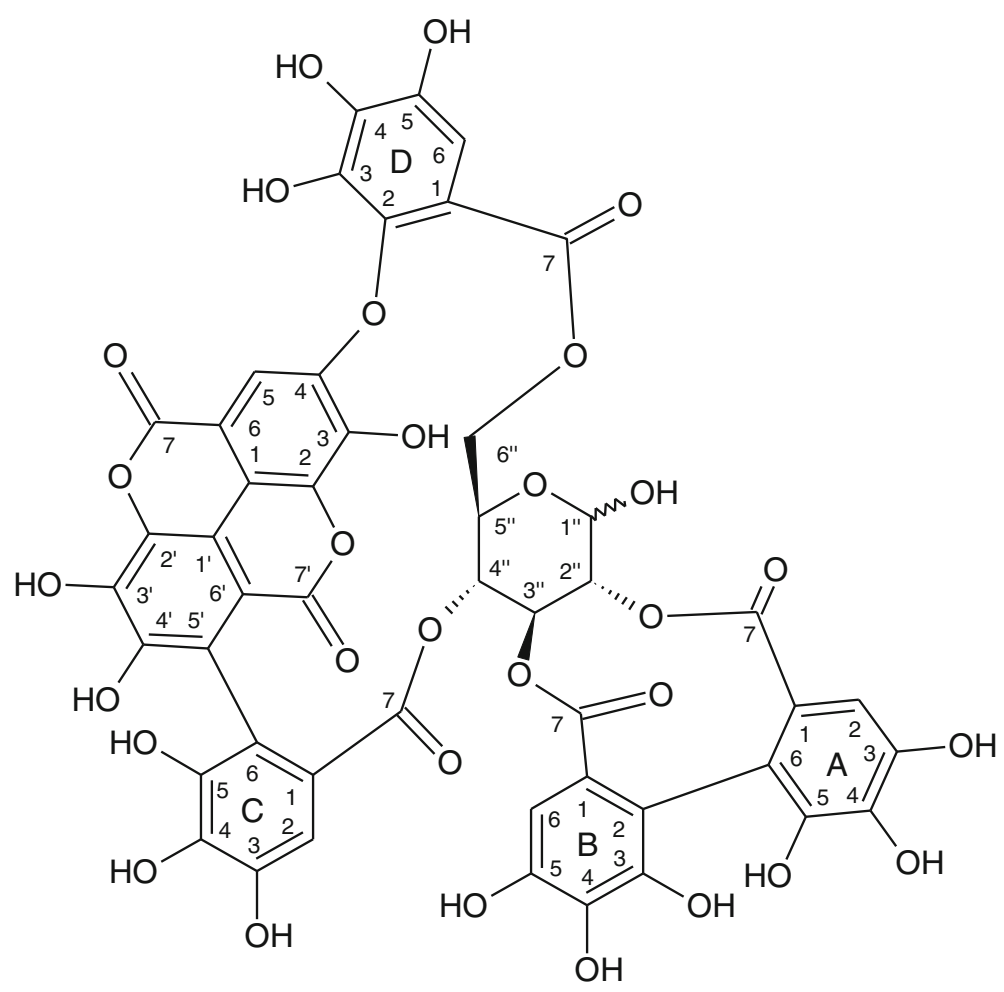

Fig. 3 Terchebulin. Tan powder. LC-MS (negative ion mode) m/z: $1083(\mathrm{M}-\mathrm{H}){ }^{1}{ }^{1} \mathrm{H}-\mathrm{NMR}$ (in $\mathrm{CD}_{3} \mathrm{OD}$ ): $\delta(\mathrm{ppm}) 3.04$ (t, $J=11.6 \mathrm{~Hz}$, one of the $\mathrm{H}-6$ "), $4.21\left(\mathrm{t}, J=10.3 \mathrm{~Hz}, \mathrm{H}-5^{\prime \prime}\right), 4.48\left(\mathrm{t}, J=8.9 \mathrm{~Hz}\right.$, one of the H-6"), $4.78\left(\mathrm{t}, J=11.0 \mathrm{~Hz}, \mathrm{H}-4^{\prime \prime}\right), 4.98\left(\mathrm{dd}, J=3.5,9.7 \mathrm{~Hz}, \mathrm{H}-2^{\prime \prime}\right), 5.23\left(\mathrm{~d}, J=2.8 \mathrm{~Hz}, \mathrm{H}-1^{\prime \prime}\right)$, $5.64\left(\mathrm{t}, J=9.6 \mathrm{~Hz}, \mathrm{H}-3^{\prime \prime}\right), 6.37(\mathrm{~s}, \mathrm{H}-\mathrm{B} 6), 6.42(\mathrm{~s}, \mathrm{H}-\mathrm{D} 6), 6.56(\mathrm{~s}, \mathrm{H}-\mathrm{A} 2), 6.79(\mathrm{~s}, \mathrm{H}-\mathrm{C} 2), 7.48(\mathrm{~s}, \mathrm{H}-5) .{ }^{13} \mathrm{C}-\mathrm{NMR}$ (in CD $\left.{ }_{3} \mathrm{OD}\right): \delta$ (ppm) $63.4\left(\mathrm{C}-6^{\prime \prime}\right), 68.5$ $\left(C-4^{\prime \prime}\right), 69.0\left(C-5^{\prime \prime}\right), 74.1\left(C-3^{\prime \prime}\right), 74.2\left(C-2^{\prime \prime}\right), 90.2\left(C-1^{\prime \prime}\right), 106.4(C-B 6), 106.5$ (C-D6), 106.8 (C-A2), 108.5(C-C2), 112.0-114.0 (C-A6, B2, 5, 5', 1, 1', 2, 2', 6, 6'), 116.0 (C-C6), 122.2 (C-D1), 123.5 (C- B1,C1), 125.1 (C-A1), 135.9 (C-B4), 136.1 (C-A4), 137.5 (C-C4), 137.6 (C-D4), 138.4 (C-3), 139.1 (C-D3), 140.7 (C-3'), 141.7 (C-D2), 143.4-143.6 (C-A5, B3, C5), 144.5-144.6 (C-A3, B5, C3, D5), 147.4 (C-4'), 150.3 (C-4), 158.3 (C-7'), 159.5 (C-7), 166.9 (C-D7), 167.0 (C-C7), 168.9 (C-A7), 169.5 (C-B7)

\section{Inhibitory activities of compounds isolated from $C$.} hartmannianium bark against $P$. gingivalis and MMP-9

In the present study, some plants belong to combretaceae family revealed good inhibitory activities against $P$. gingivalis and MMP-9; such as methanolic extracts of $T$. laxiflora, C. hartmannianium (bark) and T. brownii (bark). The potency of $T$. laxiflora was probably due to the presence of terchebulin and flavogalonic acid dilactone in wood at high concentration [15]. Kosei et al. [36] isolated gallic acid, punicalagin, terchebulin, ellagic acid 4O- $\alpha$-L-rhamnopyranoside, ellagic acid, and 3, 4, 3'-tri-Omethylellagic acid from methanolic extracts of $T$. brownii bark. However, there is no data was reported in literature regarding the isolated compounds from C. hartmannianium species bark, which makes it a potential candidate for further separation and isolation of compounds.

Among antimicrobial active compounds isolated from Combretum spp. are; combretastatins, acidic tetracyclic and pentacyclic triterpenes/triterpenoids, ellagitannins, phenanthrenes, flavonoids and saponins [37, 38]. C. hartmannianum gave good activity against Grampositive and Gram-negative bacteria, and the most of the activity was found in water and methanol extracts. Additionally, the extracts of $C$. hartmannianum were found to be active against enzymes such as reverse transcriptase and tyrosine kinase [39].

Bioassay guided fractionation led to the isolation of two compounds namely, flavogalonic acid dilactone and terchebulin (Figs. 2, 3). Terchebulin, and to a lesser extent flavogalonic acid dilactone showed combined activity against P. gingivalis and MMP-9 (Table 4). Marquis et al. [40] reported that polyphenols reduced MMP-9 activity and $P$. gingivalis growth; since polyphenols were reported to possess antimicrobial and anti-inflammatory properties, they might be of interest as therapeutic agents for controlling periodontal diseases, which involved both pathogenic bacteria and host immune responses.

Terchebulin and flavogalonic acid dilactone had moderate antibacterial activity with MIC values of 500 and $1000 \mu \mathrm{g} / \mathrm{ml}$, respectively. Previous studies showed that flavogalonic acid dilactone, terchebulin and punicalagin isolated from Terminalia spp. demonstrated antibacterial activity against $P$. acnes and Helicobacter pylori in a range between 125 to $250 \mu \mathrm{g} / \mathrm{ml}[15,41]$. Terchebulin 
Table 4 Minimum inhibitory concentration (MIC) and matrix metalloproteinases -9 (MMP-9) inhibitory activities of isolated compounds from Combretum hartmannianium bark

\begin{tabular}{lll}
\hline Compounds & MIC $(\mu \mathrm{g} / \mathrm{ml})$ & ${ }^{*}{ }^{*} C_{50}$ against MMP-9 $(\mu \mathrm{M})$ \\
\hline Terchebulin & 500 & $6.7 \pm 1.5^{\mathrm{a}}$ \\
Flavogallonic acid dilacton & 1000 & $36.1 \pm 7.5^{\mathrm{b}}$ \\
\hline
\end{tabular}

${ }^{*} \mathrm{C}_{50}$-Half minimal inhibitory concentration

Means with different letters in the same column were significantly different at the level $(p<0.05) ; n=3$

demonstrated more potent activity $(6.7 \mu \mathrm{M})$ than flavogalonic acid dilactone against MMP-9. Moreover, terchebulin has more reliable activity than chlorhexidine that inhibits MMP-9 at the $\mathrm{IC}_{50} 25.2 \mu \mathrm{M}$ [42]. Furthermore, Arabaci et al. [43] found that chlorhexidine had a few genotoxic and cytotoxic effects on human lymphocytes. Studies of the in vitro cytotoxic activity on mouse fibroblasts of terchebulin and flavogalonic acid dilactone showed activity at minimum cytotoxic concentration of $\geq 1500 \mu \mathrm{g} / \mathrm{ml}$ (1348, $3192 \mu \mathrm{M}$ respectively) [44]. To the best of our knowledge, hydrolysable tannins mainly, terchebulin and flavogalonic acid dilactone were isolated from C. hartmannianium bark for the first time during this study.

\section{Conclusions}

Our study demonstrated that some methanolic crude extracts of Sudanese medicinal plants possessed good combined activities against $P$. gingivalis and MMP-9. Moreover, this study provided new information on terchebulin and flavogalonic acid dilactone which were isolated from methanolic extracts of $C$. hartmannianium bark, indicating that they possessed interesting inhibitory properties against $P$. gingivalis and MMP-9, and this may be useful for the prevention and treatment of periodontal diseases. Further studies are recommended to investigate the mechanisms of action of these isolated compounds, toxicity and their usefulness as a source of new components in mouthwashes and toothpastes.

\section{Additional file}

Additional file 1: Table S1. IC50values $(\mu \mathrm{g} / \mathrm{ml})$ of the potent methanolic extracts of Sudanese medicinal plants against glucosyltransferase (GTFs) enzyme. (DOCX $12 \mathrm{~kb}$ )

\section{Acknowledgements}

The authors would like to thank prof. Abdelkhalig Muddathir and Dr. Farouk Hassan Eltahir for the English editing of the manuscript.

\section{Funding}

No fund was available.

Availability of data and materials Not applicable.

\section{Authors' contribution}

E.A.M.M participated in the design of the study, antibacterial, enzyme assay, isolation of compounds and write the manuscript. A.M participated in collection, extraction of plant samples and helped to draft the manuscript. T.M supervised and designs this study. All the authors read and approved the final version of the manuscript.

\section{Competing interests}

The authors declare that they have no competing interests.

Consent for publication

Not applicable.

Ethics approval and consent to participate

Not applicable.

\section{Publisher's Note}

Springer Nature remains neutral with regard to jurisdictional claims in published maps and institutional affiliations.

\section{Author details}

${ }^{1}$ Faculty of Pharmacy, University of Science and Technology, Omdurman, Sudan. ${ }^{2}$ Department of Applied Biological Science, Faculty of Applied Biological Science, Gifu University, 1-1 Yanagido, Gifu 501-1193, Japan. ${ }^{3}$ Department of Horticulture, Faculty of Agriculture, University of Khartoum, Khartoum North-Shambat, Sudan.

Received: 19 August 2016 Accepted: 8 April 2017

Published online: 20 April 2017

\section{References}

1. Socransky SS, Haffajee AD, Cugini MA, Smith C, Kent Jr RL. Microbial complexes in subgingival plaque. J Clin Periodontol. 1998;25:134-44.

2. Slots J, Bragd L, Wikstro MM, Dahlen G. The occurrence of actinobacillus actinomycetemcomitans, bacteroides gingivalis bacteroides intermedius in destructive periodontal disease in adults. J Clin periodontal. 1986;13:570-7.

3. Holt SC, Felton J, Brunsvold M, Kornman KS. Implantation of bacteroide gingivalis in non-human primate initiates progression of periodontitis. Science (WashDC). 1988;1239:55-7.

4. Schwartz Z, Goultschin J, Dean DD, Boyan BD. Mechanisms of alveolar bone destruction in periodontitis. Periodontol. 1997;14:158-72.

5. Chang YC, Lai CC, Yang SF, Chan Y, Hsieh YS. Stimulation of matrix metalloproteinases by black-pigmented bacteroides in human pulp and periodontal ligament cell cultures. J Endodon. 2002;28:90-3.

6. Chang YC, Chu SC, Yang SF, Hsieh YS, Yang LC, Huang FM. Examination of the signal transduction pathways leading to activation of gelatinolytic activity by interleukin-1 and Porphyromonas gingivalis in human osteosarcoma cells. J Periodontal Res. 2004;39:168-74.

7. Chang YC, Yang SF, Lai CC, Liu JY, Hsieh YS. Regulation of matrix metalloproteinase production by cytokines, pharmacological agents and periodontal pathogens in human periodontal ligament fibroblast cultures. J Periodontal Res. 2002;37:196-203.

8. Varghese J, Tumkur VK, Ballal V, Bhat GS. Antimicrobial effect of Anacardium occidentale leaf extract against pathogens causing periodontal disease. Adv Biosci Biotechnol. 2013;4:15-8.

9. Ohara A, Saito F, Matsuhisa T. Screening of antibacterial activities of edible plants against Streptococcus mutans. Food Sci Technol Res. 2008;14:190-3.

10. Palombo EA. Traditional plant extracts and natural products with activity against oral bacteria: potential application in the prevention and treatment of oral diseases. Evid Based Complement Alternat Med. 2011;201:1-15.

11. Patra JK, Kim ES, Oh K, Kim HJ, Kim Y, Baek KH. Antibacterial effect of crude extract and metabolites of Phytolacca americana on pathogens responsible for periodontal inflammatory diseases and dental caries. BMC Complement Altern Med. 2014;14:343.

12. Maydell HJV. Trees and Shrubs of the Sahel. Their Characteristics and Uses, (GTZ) GmbH, Germany, 1990.

13. Elegami AA, El-Nima El, El Tohami MS, Muddathir AK. Antimicrobial activity of some species of the family Combretaceae. Phytother Res. 2002;16:555-61.

14. Eldeen MS, Van Staden J. Anti-inflammatory and mycobacterial activity of leaf extracts of Coleonema album. South Afr J Bot. 2008;74:345-7. 
15. Muddathir AM, Mitsunaga T, Kosei Y. Anti-acne activity of tannin-related compounds isolated from Terminalia laxiflora. J Wood Sci. 2013;5:426-31.

16. Hirano $Y$, Kondo R, Sakai K. 5a-Reductase inhibitory tannin-related compound isolated from Shorea laevifolia. J Wood Sci. 2003:49:339-42.

17. Lin TC, Nonaka Gl, Nishioka I, Ho FC. Tannins and related compounds, CII. Structures of terchebulin, an ellagitannin having a novel tetraphenyl carboxylic acid (terchebulic acid) moiety, and biogenetically related tannins from Terminalia chebula Retz. L. Chem Pharm Bull. 1990;38:3004-8.

18. Iwaki K, Koya-Miyata S, Kohno K, Ushio S, Fukuda S. Antimicrobial activity of Polygonum tinctorium lour: extract against oral pathogenic bacteria. J Nat Med. 2006:60:121-5

19. Eloff JN. Antibacterial activity of Marula (Sclerocarya birrea (a. Rich) Hochst. Subsp. Caffra (Sond) Kokwaro) (Anacardiaceae) bark and leaves. J Ethnopharmacol. 2001;76:305-8

20. Choi JS1, Ha YM, Joo CU, Cho KK, Kim SJ, Choi IS. Inhibition of oral pathogens and collagenase activity by seaweed extracts. J Environ Biol. 2012;33:115-21.

21. Cowan MM. Plant products as antimicrobial agents. Clin Microbiol Rev. 1999;12:564-82.

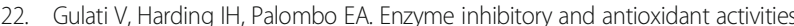
of traditional medicinal plants: potential application in the management of hyperglycemia. BMC Complement Altern Med. 2012;12:77.

23. Muddathir AM, Mitsunaga T. Evaluation of anti-acne activity of selected Sudanese medicinal plants. J Wood Sci. 2013;59:73-9.

24. Eloff $J N$, Katerel DR, McGaw $L$ J. The biological activity and chemistry of the southern African Combretaceae. J Ethnopharmacol. 2008;119:686-99.

25. Martini ND, Katerere DRP, Eloff JN. Biological activity of seven antibacterial flavonoids isolated from Combretum erythrophyllum (Combretaceae). J Ethnopharmacol. 2004;93:207-12.

26. Basile A, Giordano S, Lopez-Saez JA, Cobianchi RC. Antibacterial activity of pure flavonoids isolated from mosses. Phytochemistry. 1999;52:1479-82.

27. Eldeen IMS, Van SJ. In vitro pharmacological investigation of extracts from some trees used in Sudanese traditional medicine. South Afr J Bot. 2007;73: 435-40

28. Zanatta FB, Antoniazzi RP, Rösing CK. Staining and calculus formation after $0.12 \%$ chlorhexidine rinses in plaque-free and plaque covered surfaces: a randomized trial. J Appl Oral Sci. 2010;18:515-21.

29. Amanlou M, Beitollahi JM, Abdollahzadeh S, Tohidast-Ekrad Z. Miconazole gel compared with Zataria multiflora Boiss. Gel in the treatment of denture stomatitis. Phytother Res. 2006;20:966-9.

30. Kitano S, Irimura K, Sasaki T, Abe N, Baba A, Miyake Y, Katunuma N, Yamamoto K. Suppression of gingival inflammation induced by Porphyromonas gingivalis in rats by leupeptin. Jpn J Pharmacol. 2001:85:84-91.

31. Preshaw PM, Hefti AF, Jepsen S, Etienne D, Walker C, Bradshaw MH. Subantimicrobial dose doxycycline as adjunctive treatment for periodontitis. A review. J Clin Periodontol. 2004;31:697-707.

32. Kusumoto T, Nakabayashi T, Kida H, Miyashiro H, Hattori M, Namba T. Screening of various plant extracts used in Ayurvedic medicine for inhibitory effects on human immunodeficiency virus type 1 (HIV-1) protease. Phytother Res. 1995;9:180-4.

33. Dell'Agli M, Galli GV, Bulgari M, Basilico N, Romeo S, Bhattacharya D, Taramelli D, Bosisio E. Ellagitannins of the fruit rind of pomegranate (Punica granatum) antagonize in vitro the host inflammatory response mechanisms involved in the onset of malaria. Malar J. 2010;9:208.

34. Folgueras AR, Pendas AM, Sanchez LM, Lopez-Otin C. Matrix metalloproteinases in cancer: from new functions to improved inhibition strategies. Int J Dev Biol. 2004;48:411-24.

35. Seigler DS. Phytochemistry of Acacia-sensu lato. Biochem Syst Ecology. 2003; 31:845-73.

36. Kosei Y, Mitsunaga T, Ali MM. Screening for melanogenesis-controlled agents using Sudanese medicinal plants and identification of active compounds in the methanol extract of Terminalia brownii bark. J Wood Sci. 2016;62:285-93.

37. El Ghazali GEB, El Tohamy MS, El Egami AB. Medicinal plants of the Sudan. Part III. Sudan: Medicinal Plants of the White Nile Province Khartoum University Press; 1994.

38. Al Magboul AZ, Bashir AK, Salih AM, Farouk A, Khalid SA. Antimicrobial activity of certain Sudanese plants used in folkloric medicine: screening for antibacterial activity (V). Fitoterapia. 1988;59:57-62.

39. Ali H, König GM, Khalid SA, Wright AD, Kaminsky R. Evaluation of selected Sudanese medicinal plants for their in vitro activity against hemoflagellates, selected bacteria, HIV-1-RT and tyrosine kinase inhibitory, and for cytotoxicity. J Ethnopharmacol. 2002;83:219-28.
40. Marquis A, Genovese S, Epifano F, Grenier D. The plant coumarins auraptene and lacinartin as potential multifunctional therapeutic agents for treating periodontal disease. BMC Complement Altern Med. 2012;2:80.

41. Silva O, Viegas S, de Mello-Sampayo C, Costa MJ, Serrano R, Cabrita J, Gomes ET. Anti-Helicobacter pylori activity of Terminalia macroptera root. Fitoterapia. 2012;83:872-6.

42. Ohtsuki T, Yokosawa E, Koyano T, Preeprame S, Kowithayakorn T, Sakai S, Toida T, Ishibashi M. Quinic acid esters from Pluchea indica with collagenase, MMP-2 and MMP-9 inhibitory activities. Phytother Res. 2008;22:264-6.

43. Arabaci T, Türkez H, Çanakçi CF, Özgöz M. Assessment of cytogenetic and cytotoxic effects of chlorhexidine digluconate on cultured human lymphocytes. Acta Odontol Scand. 2013;71:1255-60.

44. Shuaibu MN, Wuyep PA, Yanagi T, Hirayama K, Tanaka T, Kouno I. The use of microfluorometric method for activity-guided isolation of antiplasmodial compound from plant extracts. Parasitol Res. 2008;102:1119-27.

45. Mossa JS, Tarig M, Mohsin A, Rafatulla S. Pharmacological studies on aerial parts of Calotropis procera. Am J Chin Med. 1991;19:223-31.

46. El Ghazali GEB, Bari EA, Bashir AK, Salih AM. Medicinal plants of Sudan part II. Medicinal plants of eastern Nuba Mountains. Khartoum: National Council for Research; 1987

47. El Ghazali GEB, El Tohami MS, El Egami AA, Abdalla WE, Galal M. Medicinal plants of the Sudan part IV. Medicinal plants of north Kordofan. Khartoum: National Council for Research; 1997.

48. El Ghazali GEB, Abdalla WE, Khalid HE, Khalafalla MM, Hamad AA. Medicinal plants of the Sudan part V. Medicinal plants of Ingassana area. Khartoum: National Council for Research; 2003.

49. El Kamali HM, El Khalifa KF. Folk medicinal plants of riverside forests of the southern Blue Nile district. Sudan Fitoterapia. 1999;70:493-7.

50. Hussein G, Miyashiro H, Nakamura N, Hattori M, Kawahata T, Otake T. Inhibitory effects of Sudanese plant extracts on HIV-1 replication and HIV-1 protease. Phytother Res. 1999;13:31-6.

51. Nour AM, Khalid SA, Kaiser M, Brun R, Abdallah WE, Schmidt TJ. The antiprotozoal activity of sixteen asteraceae species native to Sudan and bioactivity-guided isolation of xanthanolides from Xanthium brasilicum. Planta Med. 2009;75:1363-8

52. Dahab MM, Koko WS, Osman EE. In vitro antitrichomonal activity of Xanthium brasilicum vell and Argemone mexicana L different extracts. J Med Plant Res. 2011:5:151-5.

53. Rahman MA, Mossa JS, AL-Said MS, AL-Yahya MA. Medicinal plant diversity in the flora of Saudi Arabia 1: a report on seven plant families. Fitoterapia. 2004;75:149-61.

54. Kinghorn AD, Chai HB, Sung CK, Keller WJ. The classical discovery approach to defining bioactive constituent of botanicals. Fitoterapia. 2011:82:71-9.

55. Musa S, Abdelrasool F, El E, Ahmed L, Mahmoud A, Yagi S. Ethnobotanical study of medicinal plants in the Blue Nile state, South-eastern Sudan. J Med Plants Res. 2011;5:4287-97

56. Ogbazghi W, Bein E. Assessment of non-wood forest products and their role in the livelihoods of rural communities in the gash-Barka region, Eritrea. Drylands Coordination Group Report. 2006;40:26-7.

57. Mahmoud AA, Ahmed AA, El Bassuony AA. A new chlorosesquiterpene lactone from Ambrosia maritima. Fitoterapia. 1999;70:575-8.

58. Hatil HE. Medicinal plants in east and central Africa: challenges and constraints. Ethnobot leaflet. 2009:13:364-9.

59. Ferraz AC, Angelucci ME, Da Costa ML, Batista IR, De Oliveira BH, Da Cunha C. Pharmacological evaluation of ricinine, a central nervous system stimulant isolated from Ricinus communis. Pharmacol Biochem Behav. 1999; 63:367-75.

60. Holfelder MG, Steck M, Komor E, Seifert K. Ricinine in phloem sap of Ricinus communis. Phytochemistry. 1998;47:1461-3.

61. Duke JA. Medicinal plants of the bible. Owerri, NY: Trado-Medic Books; 1983

62. Mangan JL. Nutritional effects of tannins on animal feeds. Nutr Res Rev. 1988;1:209-31.

63. Louhaichi M, Salkini AK, Estita HE, Belkhir S. Initial assessment of medicinal plants across the Libyan Mediterranean coast. Adv Environ Biol. 2011;5:359-70

64. El Ghazali GEB. Medicinal plants of Sudan part I. Khartoum: Medicinal Plants of Erkawit, National Council for Research; 1986.

65. Masoko P, Gololo SS, Mokgotho MP, Eloff JN, Howard RL, Mampuru L. Evaluation of the antioxidant, antibacterial, and antiproliferative activities of the acetone extract of the roots of Senna italica (Fabaceae). Afr J Tradit Complement Altern Med. 2010;7:138-48. 
66. Hatil HE. Effect of certain medicinal plants extracts against storage pest, Tribolium castaneum Herbst. Am Eurasian J Sustain Agric. 2009;3:139-42.

67. El Kheir YM, Salih MH. Investigation of certain plants used in Sudanese folk medicine. Fitoterapia. 1980;51:143-7.

68. El Kamali HM, Khalid SA. The most common herbal remedies in Central Sudan. Fitoterapia. 1996;57:301-6.

69. Neuwinger DH. African traditional medicine. A dictionary of plant use and application. Stuttgart, Germany: Med. Pharm. Pub; 2000

Submit your next manuscript to BioMed Central and we will help you at every step:

- We accept pre-submission inquiries

- Our selector tool helps you to find the most relevant journal

- We provide round the clock customer support

- Convenient online submission

- Thorough peer review

- Inclusion in PubMed and all major indexing services

- Maximum visibility for your research

Submit your manuscript at www.biomedcentral.com/submit 